\title{
Individual cells traffic the Vasopressin 2 Receptor to their cell surface with different success
}

Eline J. Koers ${ }^{1,2,{ }^{*}, \text { Bradley A. Morgan }}{ }^{1,3,4}$, lain B. Styles ${ }^{1,3}$ and Dmitry B. Veprintsev ${ }^{1,2,{ }^{*}}$

${ }^{1}$ Centre of Membrane Proteins and Receptors (COMPARE), University of Birmingham and University of Nottingham, Midlands, UK

2Division of Physiology, Pharmacology \& Neuroscience, School of Life Sciences, University of Nottingham, Nottingham, NG7 2UH, UK

${ }^{3}$ School of Computer Science, University of Birmingham, Edgbaston, Birmingham, B15 2TT, UK and The Alan Turing Institute, London, NW1 2DB, UK

${ }^{4} \mathrm{MRC}$ IMPACT Doctoral Training Programme, Universities of Birmingham, Leicester and Nottingham, Midlands, UK

*correspondence should be addressed to Eline.Koers@nottingham.ac.uk and Dmitry.Veprintsev@nottingham.ac.uk.

\section{Summary}

G protein coupled receptors (GPCRs) translate the actions of hormones and neurotransmitters into intracellular signalling events. Mutations in GPCRs can prevent their correct expression and trafficking to the cell surface and cause disease. Single cell subcellular localisation measurements reveal that while some cells appear to traffic the majority of the vasopressin 2 receptor (V2R) molecules to the cell surface, others retain a greater number of receptors in the ER or have approximately equal distribution. Mutations in the V2R affect the proportion of cells able to send this GPCR to their cell surface but surprisingly they do not prevent all cells from correctly trafficking the mutant receptors. These findings reveal the potential for rescue of mutant receptor cell surface expression by pharmacological manipulation of the GPCR folding and trafficking machinery.

\section{Introduction}

G protein coupled receptors (GPCRs) coordinate functions of multicellular organisms by sensing hormones, neurotransmitters or cytokines that circulate around the body and inducing intracellular responses. Missense mutations in these receptors often cause loss-of-function and as such mutations are linked to 55 monogenic diseases (Schöneberg, Liebscher et al. 2020).

Missense mutations in GPCRs can lead to a change or loss of ligand binding and intracellular signalling (i.e. function), but the majority of mutations causing disease are located outside of the key functional sites -i.e. ligand binding pocket, $G$ protein and $\beta$ arrestin binding sites- (Hauser, Chavali et al. 2017) and hence are more likely to affect the folding, structure or stability of the GPCR. 
GPCRs are membrane proteins that function almost exclusively at the cell surface. Following their synthesis by ribosomes, they are inserted into the membrane of the ER using specialised protein complexes -Sec61 and EMC-(Chitwood, Juszkiewicz et al. 2018), then folded and trafficked to the cell surface.

The number of receptors at the cell surface is an important factor controlling how cells respond to signals or mediate the action of drugs. If there are no receptors, there will be no signalling in response to an increase in hormone concentration. In contrast, too much receptor means uncontrollable "always on" signalling. The number of receptors is influenced by the amount of cellular mRNA produced and the efficiency of protein biogenesis.

Vasopressin 2 Receptor (V2R) is mainly known for its function in the kidney where it promotes translocation of the aquaporin 2 channel to the apical plasma membrane of the epithelial cells of the collecting duct, resulting in water reabsorption (Wilson, Miranda et al. 2013). Many mutations in the Vasopressin 2 Receptor (V2R) are associated with NDI (nephrogenic diabetes insipidus) and cause a loss-of-function phenotype, preventing the kidneys' response to the hormone vasopressin. Several deletions and mutations have been reported to cause accumulation of the receptor in the ER (Robben, Knoers et al. 2005), while addition of the antagonist SR121463 can restore cell surface expression for some mutants (Morello, Salahpour et al. 2000). The possible mechanisms suggested to cause accumulation in the ER are misfolding, maturation defects or increased interaction with ER-resident protein calnexin (Morello 2001) (Morello, Salahpour et al. 2001)

However, the exact molecular mechanism by which these mutations trigger ER retention of V2R is still not clear while many potential effectors are involved in GPCR folding and quality control (Achour, Labbé-Jullié et al. 2008). Moreover, a dependence on the cell type with respect to the subcellular localisation of a GPCR has been reported. (Blagotinšek Cokan, Mavri et al. 2020)

We hypothesise that the interplay of a specific mutation and the cellular environment is a strong factor in determining receptor fate and biogenesis efficiency. To test this, we systematically probed a large panel of mutants for expression and localisation in the same cell type. We measured individual cell outcomes to probe variations in a homogenous cell population.

We chose to study the expression and subcellular localisation of a number of previously identified by alanine scanning mutagenesis variants of V2R with reduced cell surface expression of V2R (Heydenreich 2016) as well as a panel of NDI causing mutants.

We show that the majority of the 29 (Table S1) alanine mutations we studied lead to the retention of V2R in the ER, preventing its correct trafficking to the cell surface. The analysis of the structural context of these mutations suggest that they are likely to be folding or, more generally, biogenesis mutants because they lie outside of the functionally important ligand and $G$ protein and $\beta$-arrestin binding sites. Overall, at the cell population level, we observe a strong correlation between mutations and subcellular localisation. However, the analysis of the subcellular localisation of the V2R mutants in individual cells suggested that in some cells they are concentrated in 
the ER while in others they can traffic to the cell surface or be distributed approximately evenly between the ER and the cell surface. While the molecular basis for existence of these distinct "folding and trafficking" states of cells is not clear, it does suggest that there are some common factors that could be controlled for therapeutic purposes. Given the strong structural conservation among GPCRs, our findings are likely to be applicable to many other GPCRs where mutations cause diseases and open novel questions in the field of GPCR biogenesis.

\section{Results}

\section{Large screen of subcellular localisation of V2R mutants reveals early biogenesis defects}

We investigated whether missense mutations causing nephrogenic diabetes insipidus (NDI) are located at functional sites -i.e. ligand binding pocket, G-protein and $\beta$-arrestin binding sites- or that they predominantly located outside these functional sites and hence more likely to cause loss-of-function because of structural reasons.

The functional sites were defined using data from functional experiments using alanine scanning approach (Heydenreich 2016). We found that $30 \%$ of NDI causing missense mutations are found in functional sites and $70 \%$ outside of functional sites (Fig 1A). The expected value for a random distribution of mutations is $26 \%$ in functional sites versus $74 \%$ outside of functional sites. The observation that many SNPs in GPCRs are located outside of the functional regions is also supported for other GPCRs (Hauser, Chavali et al. 2017).

We also analysed a synthetic mutant library of V2R, an alanine scanning library. The cell surface expression rates of these mutants have been reported (Heydenreich 2016). V2R mutants with lower than $25 \%$ expression at the cell surface compared to WT V2R are shown in figure $1 \mathrm{~B}$. We found that $21 \%$ of the low surface expression causing mutations are in functional sites versus $79 \%$ outside functional sites. 

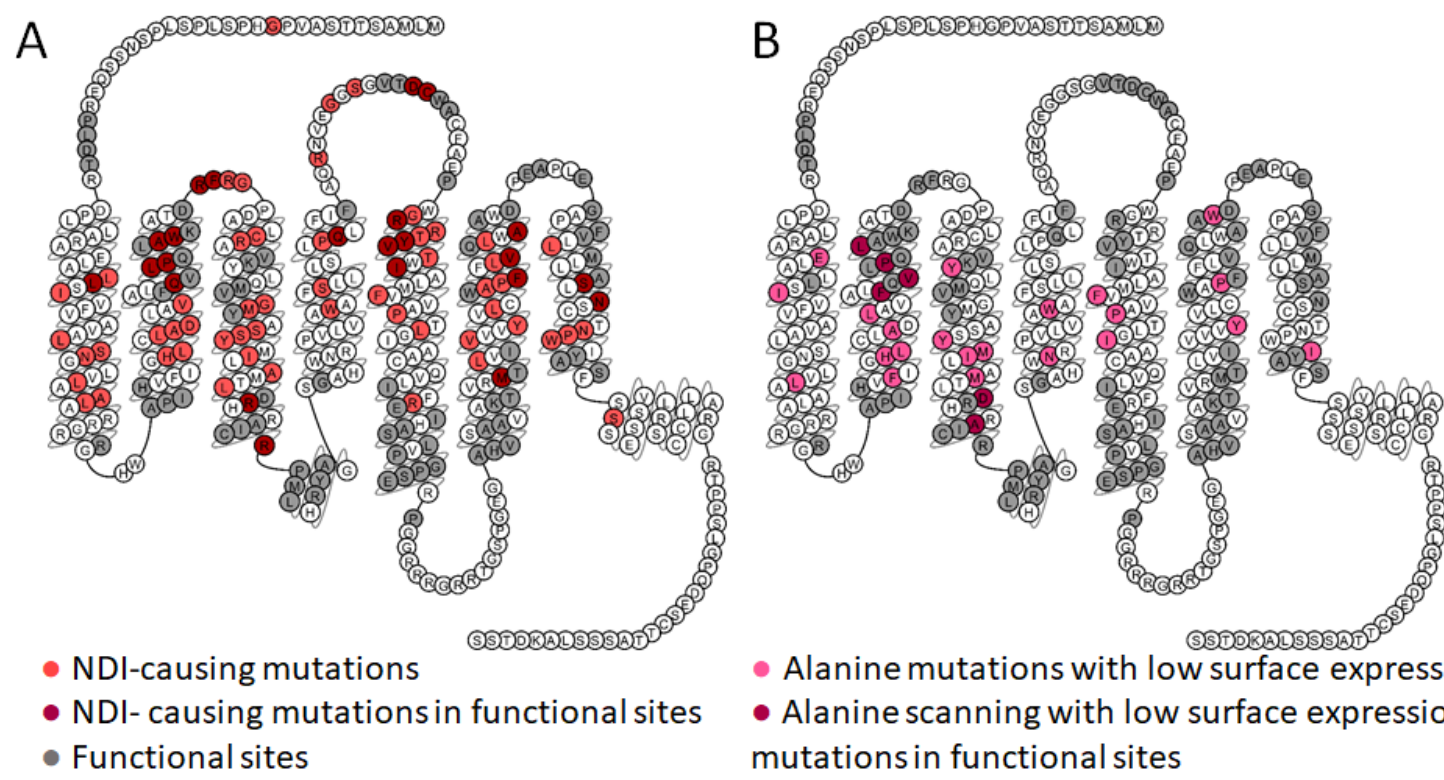

- Alanine mutations with low surface expression

- Alanine scanning with low surface expression mutations in functional sites

- Functional sites

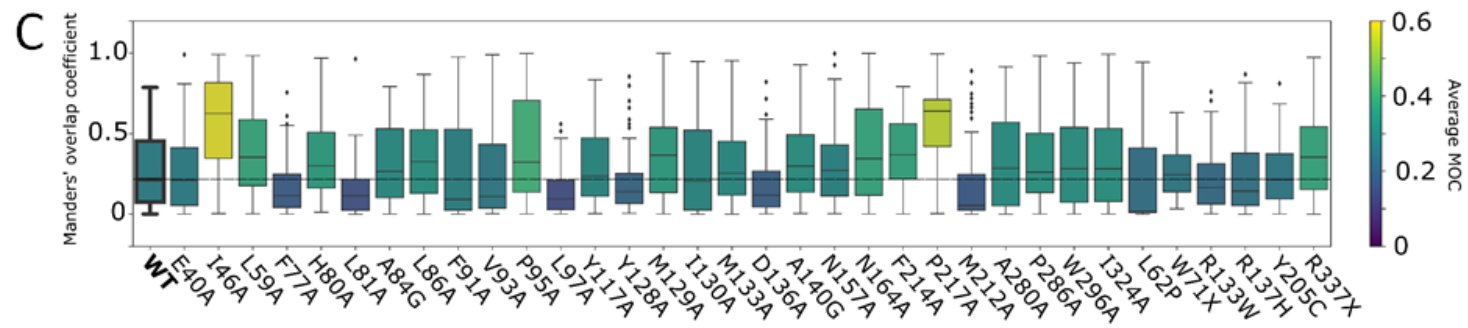

Figure 1. A) Distribution of NDI-causing mutations (Spanakis, Milord et al. 2008) shown on the snake plot of human V2R (Pandy-Szekeres, Munk et al. 2017). 30\% of NDI-causing mutations are located in the functional sites and $70 \%$ are outside. B) Distribution of alanine scanning mutants with low $(<25 \%$ WT) cell surface expression (Heydenreich 2016). 21\% in functional sites and $79 \%$ outside functional sites. C) Subcellular localisation of low expressing mutants varies between cell surface and ER. Depicted are average numbers and quantiles.

Since most of the low surface expression alanine mutants are located outside the functional sites, we wondered whether they have early biogenesis defects and are held in the ER as a result - e.g. incorrect topology, misfolding or structural instability. We utilised confocal microscopy combined with immunostaining to test this. Our results show that the majority of alanine mutants indeed show higher presence in the ER than WT pointing to early biogenesis defects as a cause for lower cell surface expression (Fig. 1C).

\section{Single cell analysis of subcellular localisation shows different rates of ER retention for cells under similar conditions}

We noticed a big spread of measured ER retention values when analysing images as a whole, as well as significant differences in subcellular localisation of V2R in individual cells (Fig 2A,B). Hence, we decided to analyse the images further on a single cell level. We set up an analysis pipeline using CellProfiler (Misteli, McQuin et al. 2018) and determined ER localisation and overall fluorescence intensity per cell. We found that the averages from whole images and per cell analysis were similar, while at the same, 
the per cell analysis shows the strong spread of ER localisation of cells grown under the same conditions expressing the same V2R mutant (fig $2 \mathrm{C}$ ).

After examining the ER localisation distribution further, we divided the cells in three categories, high, medium and low ER localisation (fig 2B). We hypothesised that cells expressing certain V2R mutants show low presence in the ER because they are overly degraded. Assuming similar mRNA quantities and stability in each cell, the lower presence of V2R could be caused by more degradation. When we examined the average fluorescence intensity we indeed saw that some mutants were present in lower amounts than WT (fig 3B). We further explored whether there was any correlation between the intensity -reflecting the protein expression level- and high ER localisation. The possibilities include high concentration of the receptor overloading the ER trafficking capability. Alternatively, low levels of protein may be linked to the efficient degradation in the ER. However, we found no correlation between V2R mutant level and ER localisation on individual cell level (fig S2). 


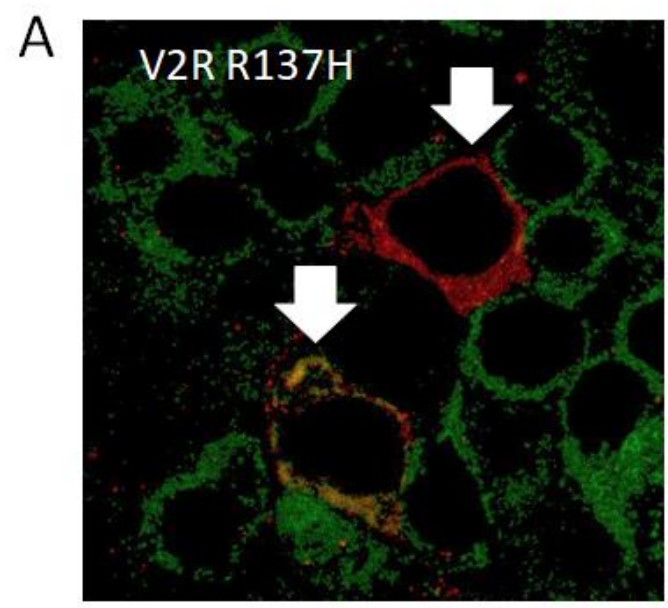

B

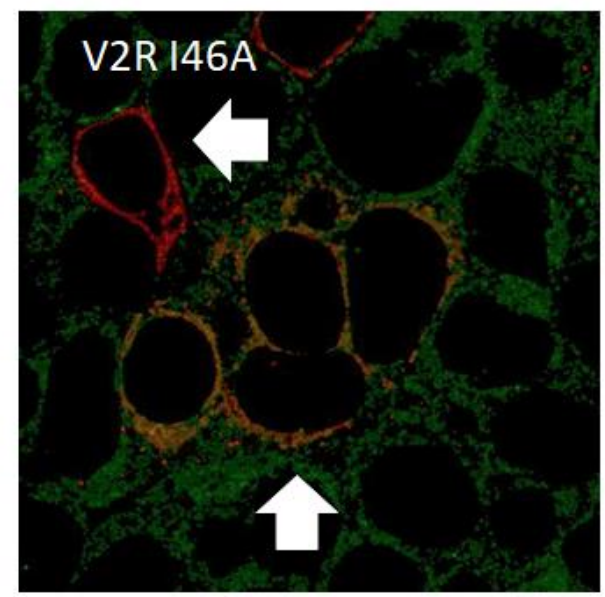

C
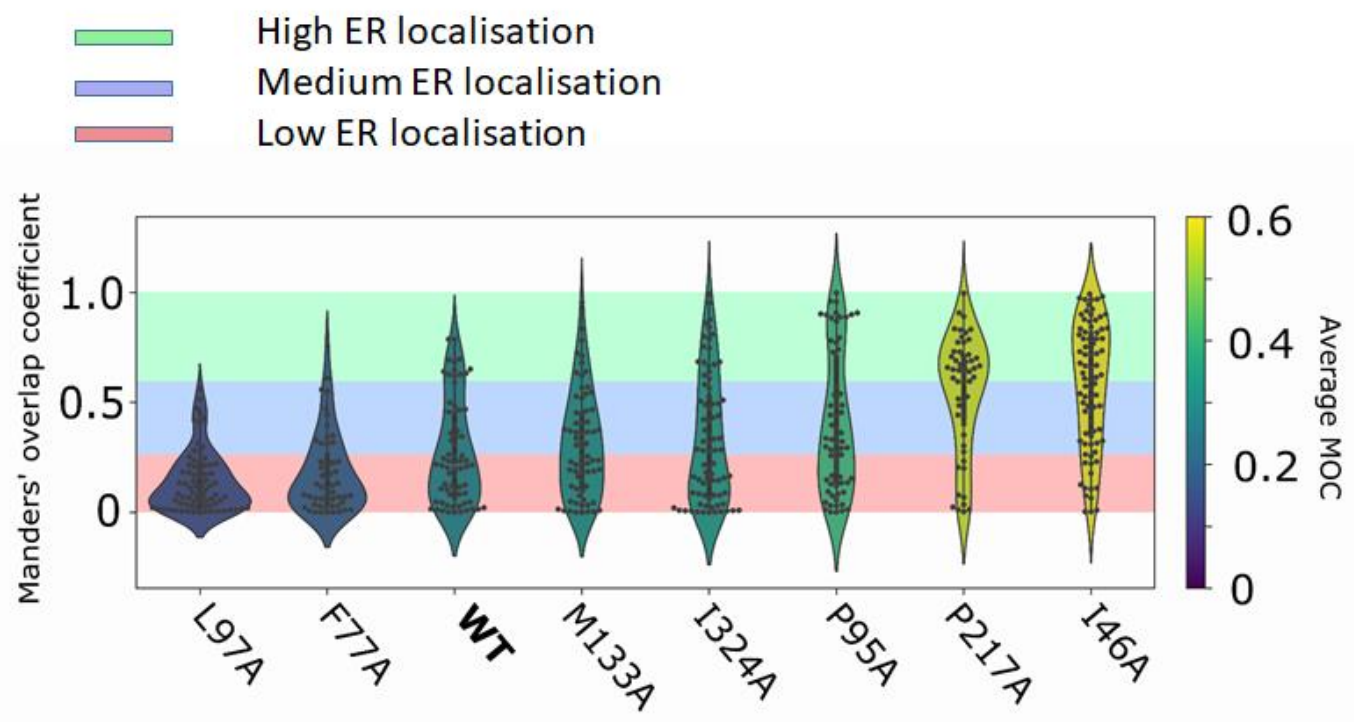

Figure $2 A, B)$ Examples of fluorescence images of cells transfected with two different V2R mutants. ER (green) and V2R (red) showing differences in ER localisation. C) ER localisation (MOC) of a selection of Alanine scanning mutations per individual cell. The cells can be divided in three categories. ER localisation data of the other alanine mutations can be found in figure SI1.

\section{V2R patient mutations show moderate presence in ER but lower overall expression}

We tested whether six common NDI-causing mutations showed an increase in ER retention compared to WT V2R. We found that, apart from a small increase for R337X, none of the others showed higher ER retention (fig $3 \mathrm{~A}$ ). We did find a weaker fluorescence intensity for all patient mutations (fig 3B, orange), which could indicate that these mutants are degraded at a higher rate. 
A
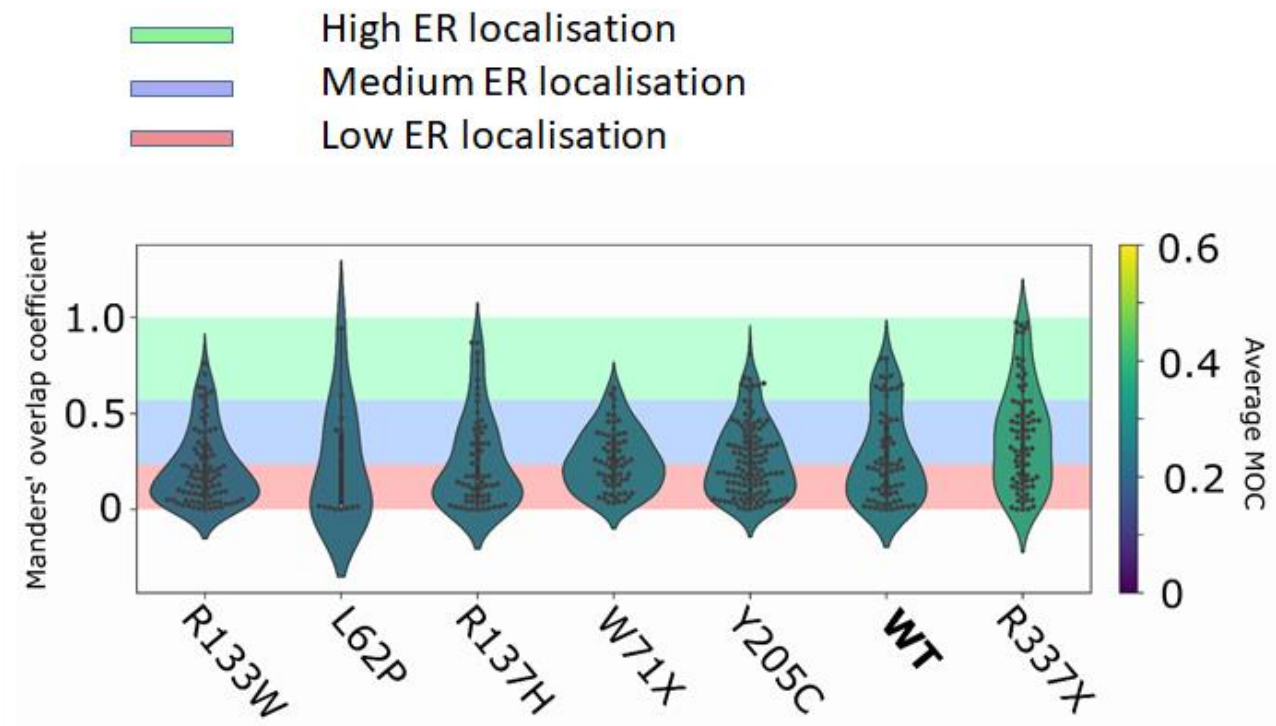

B

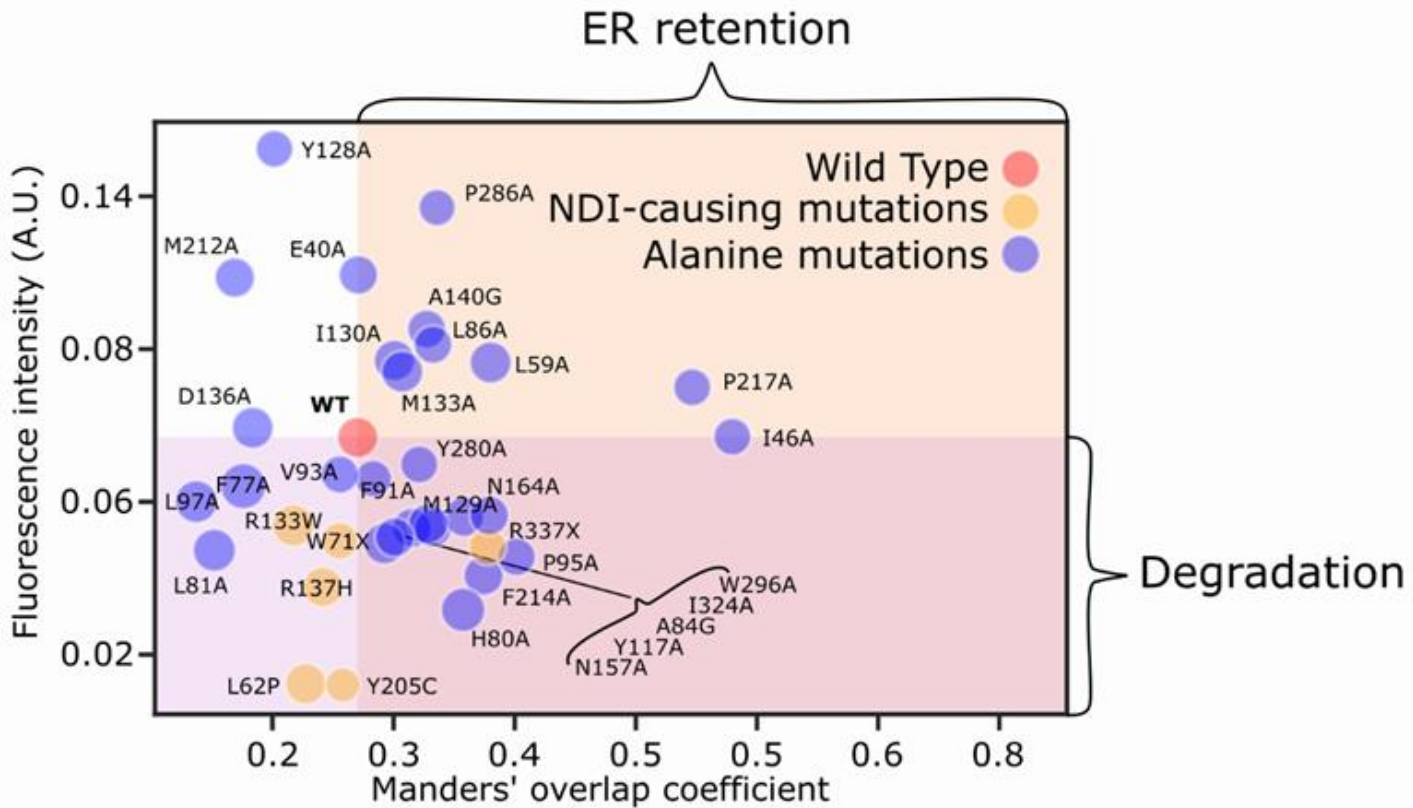

Figure 3. A) Per cell ER localisation (MOC) of NDI-causing patient mutations compared to V2R WT. All V2R patient mutations tested except V2R R337X show lower ER localisation than V2R WT, B) Average V2R expression (fluorescence intensity) and ER localisation (MOC) of all studied mutants including V2R WT (red), Alanine mutants (blue) and NDI-causing mutations (yellow). The pink area denotes lower fluorescence intensity than V2R WT which signifies lower expression levels or degradation. The orange shaded area marks higher ER localisation than V2R WT which signifies ER retention. 


\section{Discussion}

Here, we show the potential of mutations in V2R to affect the subcellular distribution of protein and cause ER retention. Mutations also affect the level of protein expression. Unexpectedly, individual cells expressing V2R and its mutants can be divided into subclasses. Some are able to express proteins normally and traffic receptors to the cell surface. Others retain receptors in the ER, and a third subclass shows the receptor distributed between the ER and cell surface. We observed this for the WT protein as well as for mutants, however the relative proportions of these sub-classes varies. We also observed that the level of expression of individual mutants also varied on a per cell basis, as well as per population.

Where could this variability of protein level and subcellular localisation originate from? A number of mRNA molecules and correspondingly, polypeptide chain production per cell can vary from cell to cell, resulting in variations in protein abundance. A common concept of "ER jamming" suggests that high levels of protein expression may lead to saturation of ER folding and trafficking machinery, and lead to increased ER retention. However, we found no correlation between protein expression level and ER retention at single cell level, suggesting that at least under our experimental conditions we have not overloaded the ER.

This leaves options of yet unidentified factors affecting the efficiency of processes of membrane insertion and folding, or quality control (QC), degradation machinery, or trafficking machinery. The protein abundance of these factors may also vary cell-tocell, resulting in these different "folding and trafficking" sub-classes. Possible analogy with the functioning of the much better understood soluble folding processes can help us to generate future hypotheses to investigate. In the case of the Hsp90/70 chaperone system, FANCA proteins with severe and function-disruptive mutations are more likely to be associated with Hsp70 while more mild function-permissive mutants are associated with Hsp90.(Karras, Yi et al. 2017) Whether a similar functional division is present in membrane proteins remains to be studied.

The NDI-causing V2R mutants we probed did not show more ER localisation compared to the WT V2R. This is in contrast with previous reports (Morello, Salahpour et al. 2000). However, the construct used here contains a soluble $\mathrm{N}$-terminal domain -a SNAP tag- which could influence the insertion pathway of GPCRs depending on V2R's dependency of insertion by EMC (Chitwood, Juszkiewicz et al. 2018).

One of the direct consequences of our observations is that the otherwise identical cells in tissues would have different levels of cell surface receptors, and will respond differently to changes in hormone and drug concentrations. The importance of this for whole tissue pharmacological response is not clear but should be considered in future studies. The other exciting aspect of this work is the finding that even rather disruptive mutants can be produced and trafficked to the cell surface suggesting that the process of biogenesis can be manipulated by targeting proteins involved in this process to increase overall production of the mutant receptor, resulting in (partial) restoration of its function. However, the next challenge is to identify the key players in GPCR biogenesis that affect the efficiency of this process. 


\section{Acknowledgements}

We thank Jeremy Pike, Clare Harwood and David Sykes for valuable advice and critical discussions and Tim Self, Seema Bagia and Robert Markus from the SLIM Imaging facility at the University of Nottingham for their support. BAM is funded by the MRC IMPACT doctoral programme. EK was funded by the IBSA foundation for scientific research.

\section{Methods}

\section{Cloning and selection of V2R mutants}

All mutants were cloned into a pcDNA4/TO vector containing an N-terminal Twin-Strep and SNAP tag (Heydenreich, Miljus et al. 2017). Alanine scanning mutant plasmids were made and obtained from (Heydenreich 2016) as well as cell surface expression data to select alanine mutants with $<25 \%$ cell surface expression compared to V2R WT. Patient mutations were cloned using a two-fragment approach (Heydenreich, Miljus et al. 2017). The selected mutants are listed in Table S1.

\section{V2R mutant expression and immunolabeling}

HEK293T cells were grown in 6-well plates with $22 \mathrm{~mm}$ square no. 1.5 coverslips coated with poly-D-lysine hydrobromide (Sigma-Aldrich). Cells in each well were transiently transfected using $100 \mathrm{ng}$ V2R and $900 \mathrm{ng}$ salmon sperm DNA and 1:3 Polyethylenimine. The cells were grown for $20-28 \mathrm{~h}$. The cells were fixed using $3 \%$ paraformaldehyde, incubated for 20 min, washed 2x with PBS. Coverslips were then elevated from 6 -well plates and cells permeabilised with $0.5 \%$ IGEPAL in PBS for 5 min at 4 , washed $2 \times 5$ min with PBS, then blocked with $3 \%$ BSA-1\% glycine in PBS, incubated for 30 min, washed 2x 5 min with PBS, then blocked with $10 \%$ Goat serum (Abcam), incubated $30 \mathrm{~min}$, then added polyclonal anti-SNAP antibody (ThermoFisher) 1:100 and Anti-P4HB antibody [RL90] (abcam) 1:500 in 10\% Goat serum and incubated overnight at $4 \mathrm{C}$. Washed coverslip $3 \mathrm{x}$ in PBS and incubated cover slips in goat-anti-rabbit-AF-647 (Abcam) 1:500, goat-anti-mouse-AF-488 (Abcam) 1:500, DAPI stain $5 \mathrm{mg} / \mathrm{mL} \mathrm{1:1000} \mathrm{in} \mathrm{10 \%} \mathrm{Goat} \mathrm{serum} \mathrm{in} \mathrm{the} \mathrm{dark} \mathrm{for} 1 \mathrm{~h}$. Washed coverslips $3 \times 5$ min with PBS and mounted coverslips with Vectashield antifade mounting medium (Vectorlabs).

\section{Microscopy}

Images were recorded using a Zeiss LSM 710 laser scanning confocal microscope fitted with a Zeiss Plan-Apochromat 63x/1.40 NA oil immersion objective. A Diode 405 $\mathrm{nm}$, Helium Neon $633 \mathrm{~nm}$ laser and Argon laser at $488 \mathrm{~nm}$ were used to excite DAPI, Alexafluor 647 and Alexafluor 488 fluorophores respectively and emission was collected using a 488/561/633 multi beam splitting filter. Images were taken at $512 \times$ 512 pixels per frame and a slice of 53.36 um for confocal images. Laser power and gain were kept constant between experiments. 


\section{Image analysis}

Whole images were analysed using Fiji (Schindelin, Arganda-Carreras et al. 2012) and a batch processing script using $1 \sigma$ Gaussian blur, Moments automatic thresholding algorithm and the Coloc 2 plugin to calculate the Manders' overlap coefficient per image.

\section{Single cell image analysis}

Single cell image analysis was done using a custom analysis pipeline in CellProfiler 3.1.9 (Misteli, McQuin et al. 2018). First, nuclei were detected based on DAPI fluorescence images, then the ER and V2R fluorescence images were automatically thresholded using the Otsu algorithm and assigned to a nuclei using a Watershed Image algorithm. Then the nucleus area was subtracted and co-localisation was measured by calculating the Manders' overlap coefficient. In addition, total intensity and area of each cell were calculated.

\section{References}

Achour, L., C. Labbé-Jullié, M. G. H. Scott and S. Marullo (2008). "An escort for GPCRs: implications for regulation of receptor density at the cell surface." Trends in Pharmacological Sciences 29(10): 528-535.

Blagotinšek Cokan, K., M. Mavri, C. S. Rutland, S. Glišić, M. Senćanski, M. Vrecl and V. Kubale (2020). "Critical Impact of Different Conserved Endoplasmic Retention Motifs and Dopamine Receptor Interacting Proteins (DRIPs) on Intracellular Localization and Trafficking of the D2 Dopamine Receptor (D2-R) Isoforms." Biomolecules 10(10).

Chitwood, P. J., S. Juszkiewicz, A. Guna, S. Shao and R. S. Hegde (2018). "EMC Is Required to Initiate Accurate Membrane Protein Topogenesis." Cell 175(6): 1507-1519.e1516.

Hauser, A. S., S. Chavali, I. Masuho, L. J. Jahn, K. A. Martemyanov, D. E. Gloriam and M. M. Babu (2017). "Pharmacogenomics of GPCR Drug Targets." Cell.

Heydenreich, F. M. (2016). The inner workings of a GPCR: molecular basis of biased G protein activation and beta-arrestin recruitment. PhD, ETH Zurich.

Heydenreich, F. M., T. Miljus, R. Jaussi, R. Benoit, D. Milic and D. B. Veprintsev (2017). "Highthroughput mutagenesis using a two-fragment PCR approach." Sci Rep 7(1): 6787.

Karras, G. I., S. Yi, N. Sahni, M. Fischer, J. Xie, M. Vidal, A. D. D’Andrea, L. Whitesell and S. Lindquist (2017). "HSP90 Shapes the Consequences of Human Genetic Variation." Cell 168(5): 856-866.e812.

Misteli, T., C. McQuin, A. Goodman, V. Chernyshev, L. Kamentsky, B. A. Cimini, K. W. Karhohs, M. Doan, L. Ding, S. M. Rafelski, D. Thirstrup, W. Wiegraebe, S. Singh, T. Becker, J. C. Caicedo and A. E. Carpenter (2018). "CellProfiler 3.0: Next-generation image processing for biology." PLOS Biology 16(7).

Morello, J.-P., A. Salahpour, A. Laperrière, V. Bernier, M.-F. Arthus, M. Lonergan, U. PetäjäRepo, S. Angers, D. Morin, D. G. Bichet and M. Bouvier (2000). "Pharmacological chaperones rescue cell-surface expression and function of misfolded V2 vasopressin receptor mutants." Journal of Clinical Investigation 105(7): 887-895. 
Morello, J.-P., A. Salahpour, U. E. Petäjä-Repo, A. Laperrière, M. Lonergan, M.-F. Arthus, I. R. Nabi, D. G. Bichet and M. Bouvier (2001). "Association of Calnexin with Wild Type and Mutant AVPR2 that Cause Nephrogenic Diabetes Insipidust." Biochemistry 40(23): 6766-6775.

Pandy-Szekeres, G., C. Munk, T. M. Tsonkov, S. Mordalski, K. Harpsoe, A. S. Hauser, A. J. Bojarski and D. E. Gloriam (2017). "GPCRdb in 2018: adding GPCR structure models and ligands." Nucleic Acids Res.

Robben, J. H., N. V. A. M. Knoers and P. M. T. Deen (2005). "Characterization of vasopressin V2 receptor mutants in nephrogenic diabetes insipidus in a polarized cell model." American Journal of Physiology-Renal Physiology 289(2): F265-F272.

Schindelin, J., I. Arganda-Carreras, E. Frise, V. Kaynig, M. Longair, T. Pietzsch, S. Preibisch, C. Rueden, S. Saalfeld, B. Schmid, J.-Y. Tinevez, D. J. White, V. Hartenstein, K. Eliceiri, P. Tomancak and A. Cardona (2012). "Fiji: an open-source platform for biological-image analysis." Nature Methods 9(7): 676-682.

Schöneberg, T., I. Liebscher and P. Insel (2020). "Mutations in G Protein-Coupled Receptors: Mechanisms, Pathophysiology and Potential Therapeutic Approaches." Pharmacological Reviews 73(1): 89-119.

Spanakis, E., E. Milord and C. Gragnoli (2008). "AVPR2 variants and mutations in nephrogenic diabetes insipidus: review and missense mutation significance." Journal of cellular physiology 217(3): 605-617.

Wilson, J. L. L., C. A. Miranda and M. A. Knepper (2013). "Vasopressin and the regulation of aquaporin-2." Clinical and Experimental Nephrology 17(6): 751-764.

\section{Supplementary information}

Table S1

List of the 28 Alanine scanning mutants and 6 patient mutations studied 
bioRxiv preprint doi: https://doi.org/10.1101/2021.08.09.455709; this version posted August 9, 2021. The copyright holder for this preprint (which was not certified by peer review) is the author/funder, who has granted bioRxiv a license to display the preprint in perpetuity. It is made available under aCC-BY 4.0 International license.

\begin{tabular}{|l|l|}
\hline Alanine scanning mutants & Functional site \\
\hline E40A & No \\
\hline I46A & No \\
\hline L59A & No \\
\hline F77A & No \\
\hline H80A & No \\
\hline L81A & No \\
\hline A84G & No \\
\hline L86A & No \\
\hline F1A & Yes \\
\hline V93A & Yes \\
\hline P95A & Yes \\
\hline L97A & Yes \\
\hline Y117A & No \\
\hline Y128A & No \\
\hline M129A & No \\
\hline I130A & No \\
\hline M133A & No \\
\hline D136A & Yes \\
\hline A140G & Yes \\
\hline N157A & No \\
\hline W164A & No \\
\hline F214A & No \\
\hline P217A & No \\
\hline I221A & No \\
\hline Y280A & No \\
\hline P286A & No \\
\hline W296A & No \\
\hline I324A & No \\
\hline NDI-causing mutations & \\
\hline L62P & Yes \\
\hline W71X & No \\
\hline R113W & \\
\hline R137H & Y205C \\
\hline R337X & \\
\hline
\end{tabular}



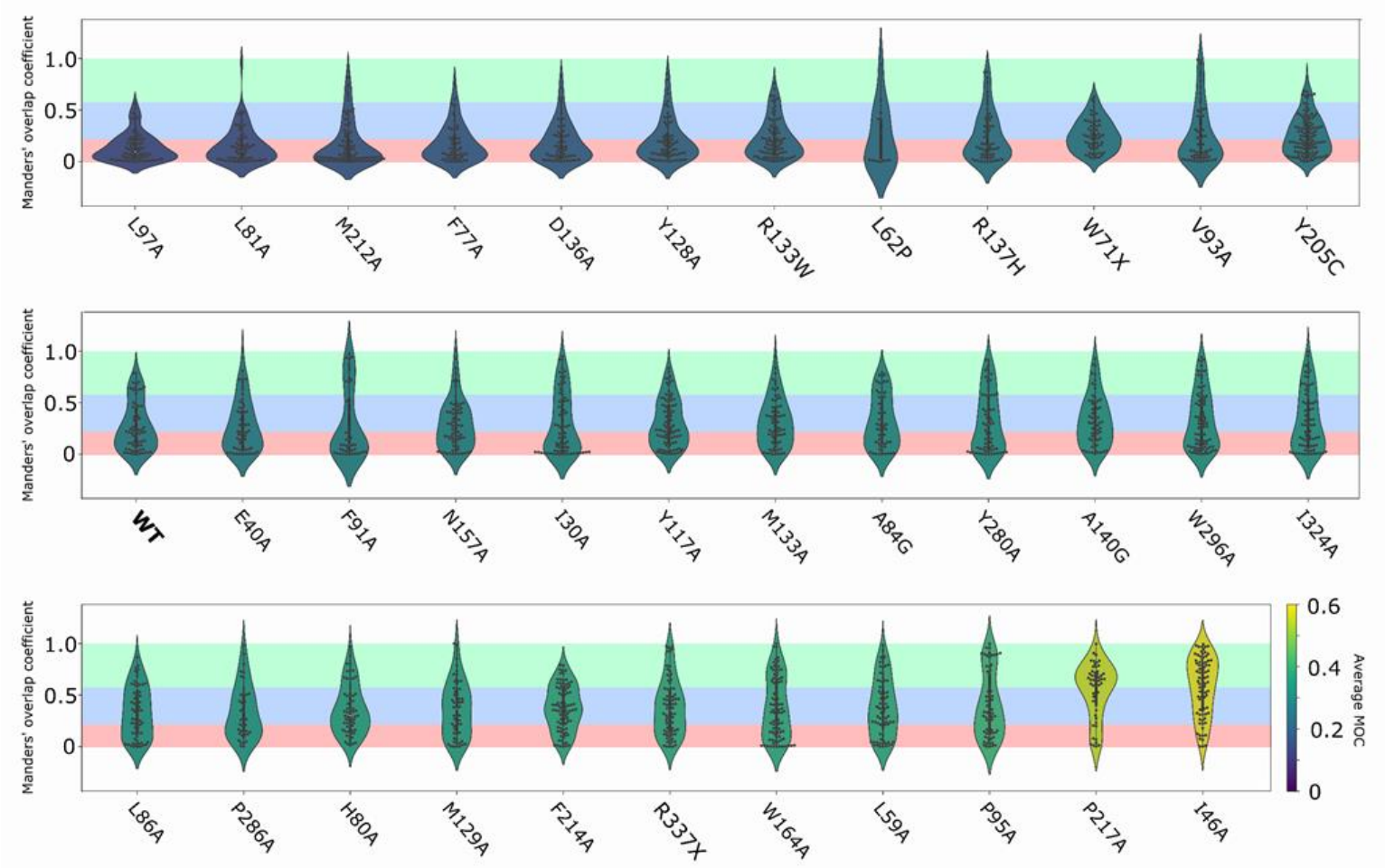

Figure S1: ER localisation per cell for all studied mutants sorted by average ER localisation. Green: high ER localisation, blue: medium ER localisation, red: low ER localisation
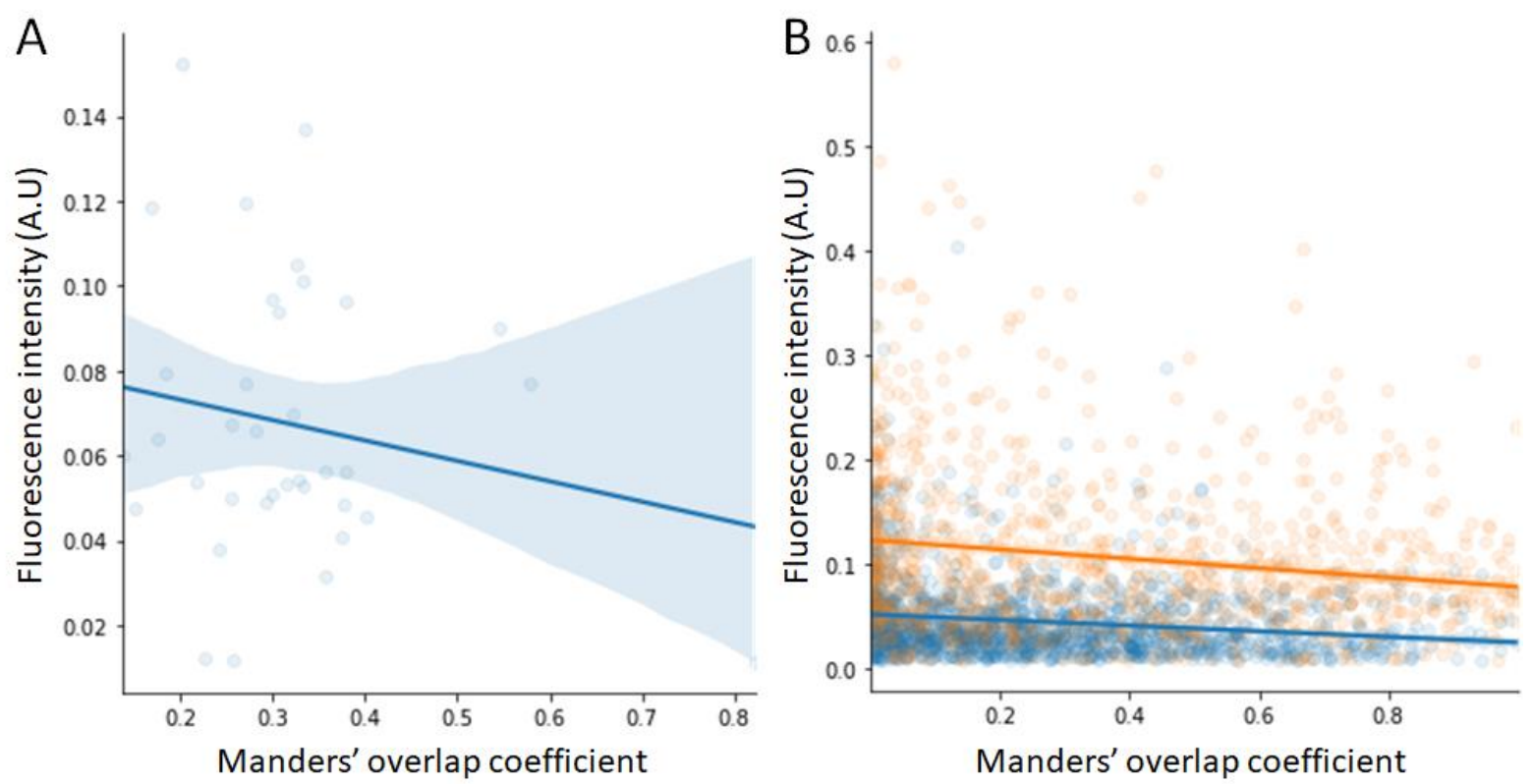

Figure S2: Correlation of V2R Fluorescence Intensity and Manders overlap coefficient. A) Average Manders overlap coefficient (MOC) and intensity per mutant $\left(R^{\wedge} 2=-4\right)$. B) MOC and intensity per cell split out per biological replicate $\left(N=2, R^{\wedge} 2=-0.9\right.$ and -1 for red and blue respectively). 
bioRxiv preprint doi: https://doi.org/10.1101/2021.08.09.455709; this version posted August 9, 2021. The copyright holder for this preprint (which was not certified by peer review) is the author/funder, who has granted bioRxiv a license to display the preprint in perpetuity. It is made available under aCC-BY 4.0 International license. 